\title{
PENTAZOCINE ABUSE INDUCED FIBROMYOSITIS
}

Sundar S1, Muthuraj K², Nagarajan V33, Ameen Azad ${ }^{4}$

\section{HOW TO CITE THIS ARTICLE:}

Sundar S, Muthuraj K, Nagarajan V, Ameen Azad. "Pentazocine Abuse Induced Fibromyositis". Journal of Evolution of Medical and Dental Sciences 2014; Vol. 3, Issue 30, July 28; Page: 8317-8321,

DOI: $10.14260 /$ jemds/2014/3048

ABSTRACT: Pentazocine is a synthetically prepared opioid analgesic drug of benzomorphan class, used to treat moderate to severe pain. As with all opioids, Pentazocine also has abuse potential. When abused, it can lead to lot of complications like skin fibrosis, skin ulceration, abnormal skin pigmentation and fibromyositis. We report a case of Pentazocine abuse induced fibromyositis in a 40 year old lady who used it for her insomnia for 11 years. She had severe diffuse fibromyositis from shoulder to ankle resulting in significant lifelong disability and bedridden state. This case emphasizes the significance of the vigilance needed in pharmaceutical industry especially in India while dispensing opioid analgesics, as almost all the cases of Pentazocine abuse induced complications were reported only from India after 1985.

KEYWORDS: Pentazocine, Drug abuse, fibromyositis, woody induration, painless contractures.

KEYMESSAGES: Pentazocine abuse can lead to debilitating complications like contractures and fibromyositis. Any patient presenting with painless contractures should be evaluated for drug abuse. General practitioner and dispensing pharmacist should be extremely cautious in delivering the injection to the patients.

INTRODUCTION: Pentazocine, belonging to benzomorphan series was the first mixed agonistantagonist opioid analgesic to be marketed..$^{1}$ It is an agonist at kappa and sigma opioid receptors and a weak antagonist at mu receptor. ${ }^{1}$ It is indicated for the relief of moderate to severe pain and also used as a supplement to surgical anesthesia. All opioid analgesics have abuse and addiction potential. Likewise Pentazocine, if abused may lead to lot of complications like skin fibrosis, skin ulceration, abnormal skin pigmentation and fibromyositis. ${ }^{2-4}$

Fibromyositis not only affect the injected areas but also in areas distal to injected sites.5, 6 We report here a 40 year old lady with diffuse severe fibromyositis due to Pentazocine abuse. Our patient also had fibromyositis in non-injected areas.

CASE HISTORY: 40 year old lady presented to our neurology OPD with the complaints of difficulty in using her both upper limbs for 6 years and both lower limbs for 2 years with inability to walk independently for one year. She also complained of induration and stiffness of muscles in lower limb predominantly around the hip. Two years back, her walking was made more difficult by a poorly treated fracture hip which she sustained due to an accidental fall. She also gave history of multiple abscesses in buttocks and thighs for the past three years.

Inspection of the extremities revealed woody induration of glutei, quadriceps and deltoid. Multiple depressed scars with surrounding hyperpigmentation were seen on skin surrounding thighs, buttocks and shoulders. In upper limbs, her shoulders were fixed in abduction at 30 degrees and elbows were semi flexed with no more than 20 degree range of movement (figure 1a). In lower limbs, both hip and knee joints were fixed in extension resulting in a peculiar gait (figure 1b). 
Distal joints were normal. Muscle power was normal within the limited range of movements. Sensations were normal.

On further enquiry with her mother, a medical practitioner, it was found out that the patient had depression and insomnia 11 years back. To overcome them, she started to inject herself daily. Frequency of injections increased from $30 \mathrm{mg}$ once daily to twice daily and later three times a day. Site of injection changed from deltoid region (Left > Right) to thigh and buttocks region once she developed contractures in the arms. She stopped taking the injections only after getting severe restrictions in her mobility for the past one year.

Investigations revealed normal hemogram, normal liver \& renal function tests. Roentgenogram of knee joints did not show any joint contractures (figure 2a). Roentgenogram of hip joints showed head and neck of femur were absorbed with narrow hip joint on right and old fracture neck of femur with wandering acetabulum on the left (figure 2b). Serum Creatine phosphokinase, calcium, phosphorus, alkaline phosphatase were normal.

Skin biopsy showed atrophic epidermis and dermis with increased collagen deposition. Dermis also showed perivascular lymphocytic infiltration. Electromyography of left deltoid \& left quadriceps showed myopathic pattern. Muscle biopsy showed interstitial \& perimysial lymphocytic infiltrate (figure 3). There was focal dense fibrosis entrapping muscle fibers along with granuloma formation which was compatible with fibromyositis (figures 4).

Differential diagnosis at the time of admission was Myositis ossificans, Stiff Person syndrome, Emery Dreifuss muscular dystrophy and infiltrative myopathies. Myositis ossificans was unlikely since the patient had no histological osteoid component and no imaging correlation. Stiff Person syndrome was less likely since the patient had no spasms or cramps. Absence of cardiac and neck involvement made the diagnosis of Emery Dreifuss muscular dystrophy unlikely.

Negative biopsy ruled out infiltrative myopathies. In view of peculiar presentation with the history of chronic pentazocine abuse, woody induration of muscles with normal joint and positive biopsy, the diagnosis of Pentazocine abuse induced fibromyositis was made.

She was placed on a vigorous physiotherapy program with passive and active stretching exercises along with gait training. At 3 months of follow up she had no appreciable change in her deformity.

DISCUSSION: The exact mechanism of pentazocine induced fibromyositis is not known. It is well soluble in acidic medium and its crystals get precipitated easily in a neutral or slightly alkaline medium inducing an inflammatory response. ${ }^{2}$ Repeated intramuscular injections will not only injure the muscle fibre but also decrease the capacity for regeneration of sarcolemma and endomysial sheath which are already disrupted by repeated injections leading to focal necrosis. ${ }^{7,8}$ Also the large volume of the injected drug will further compress the already necrosed muscle as well as the nearby capillaries resulting in muscle ischemia. Recurrent similar episodes will change the muscle into fibrosis. ${ }^{7,8,9}$

Complications of pentazocine abuse have been well documented since 19712,3,4. Our patient had all the complications of pentazocine abuse including skin induration, pigmentation, contractures, ulceration and fibromyositis. Fibromyositis was not only present in the injected muscle but also in the muscle groups which were not injected with Pentazocine. This is in line with similar cases reported previously.5, 6 
The possible mechanism could be a direct action of the drug once it enters into the circulation or the release of an independent factor from the site of injection that leads to widespread involvement of the muscles.5, 6

Our patient had severe and diffuse fibromyositis and contractures from shoulder to ankle which made her crippled and lifelong dependent on others. She used the old prescriptions of pentazocine and was able to get the drugs regularly. Unless strict action and surveillance of pharmacies are undertaken, cases like our case cannot be prevented. Almost all the cases of pentazocine abuse induced complications after 1985 are reported from India. This shows the laxness in dispensing of scheduled drugs in India.

In conclusion, this case is reported for its rarity. Whenever any patient presents with contractures without significant orthopedic disease, drug abuse as etiology should be seriously entertained. While evaluating the patients who are closely related to medical or paramedical professionals and patients with depression, drug abuse history has to be sought in detail. Pharmacies should take extra care when dispensing a scheduled drug and practitioners should take extra care while prescribing an addiction forming drug.

\section{REFERENCES:}

1. Tripathi KD, Drugs acting in Central Nervous system. In: Tripathi KD, editor. Essentials of Medical Pharmacology, 6 ${ }^{\text {th }}$ ed, New Delhi: Jaypee Publishers; 2008. P 467.

2. Schlicher JE, Zuehlke RL, Lynch PJ. Local changes at the site of pentazocine injection. Arch Dermatol 1971; 104: 90-1.

3. Winfield JB, Greer K. Cutaneous complications of parenterally administered pentazocine. JAMA 1973; 226: 189-90.

4. Steiner JC, Winkelman AC, De Jesus PV Jr. Pentazocine-induced myopathy. Arch Neurol 1973; 28: $408-9$.

5. Goyal V, Chawla JM, Balhara YP, Shukla G, Singh S, Behari M. Calcific myofibrosis due to pentazocine abuse: A case report. J Med Case Rep 2008; 2: 160.

6. Zanzmera P, Somasekharan M, Srivastava A. Myofibrosis in a pentazocine addict. Indian J Pain 2013; 27: 185-8.

7. Das CP, Thussu A, Prabhakar S, Banerjee AK. Pentazocine-induced fibromyositis and contracture. Postgrad Med J 1999; 75: 361-2.

8. Engel W K. Focal myopathic changes produced by electromyographic and hypodermic needles. "Needle myopathy". Arch Neurol 1967; 16: 509-11.

9. T. K. Shanmugasundaram. Post injection fibrosis of skeletal muscle: A clinical problem. International orthopedics 1980; 4: 31-37. 


\section{CASE REPORT}

1a): Abnormal posture of the patient and contractures at elbows and hip.

1b): Right thigh of the patient showing induration and hyperpigmentation of skin along with deformity in the knee.

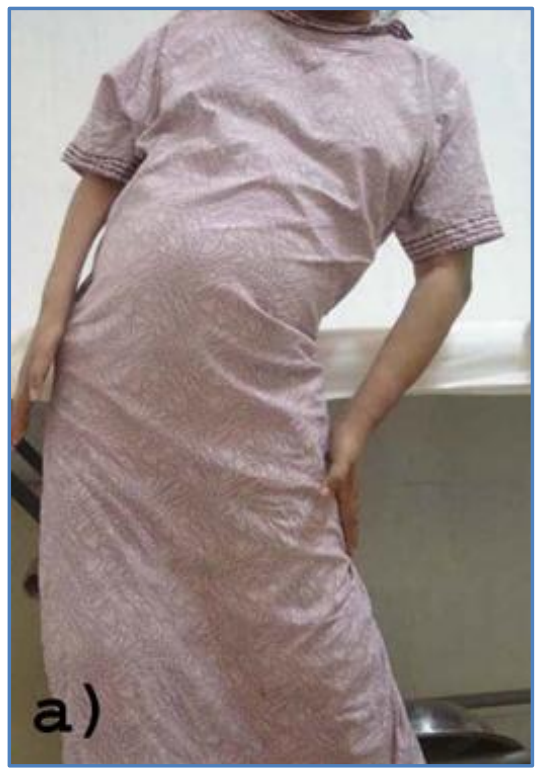

Figure 1a

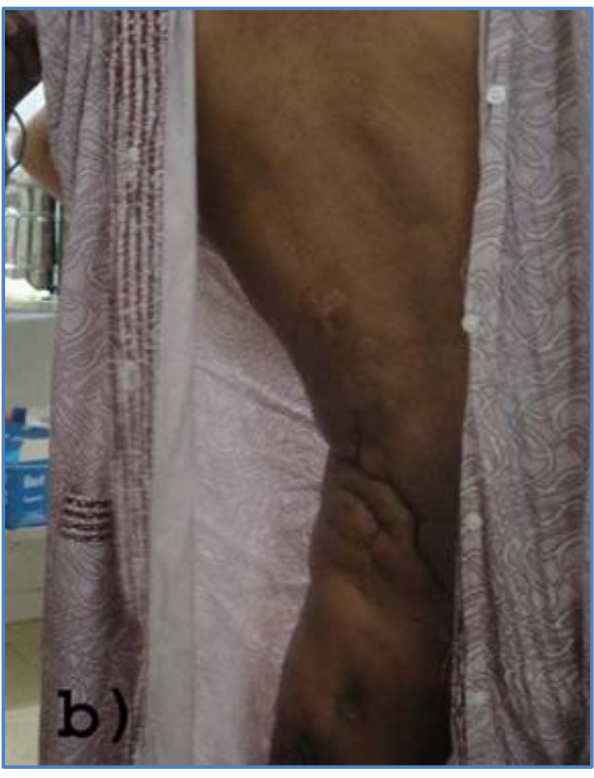

Figure 1b

2a): $X$ ray of right knee joint of the patient show normal joint space without any bony deformities.

2b): X ray of hip joint of the patient shows resorption of head of femur on the right side and subluxation of left hip joint.

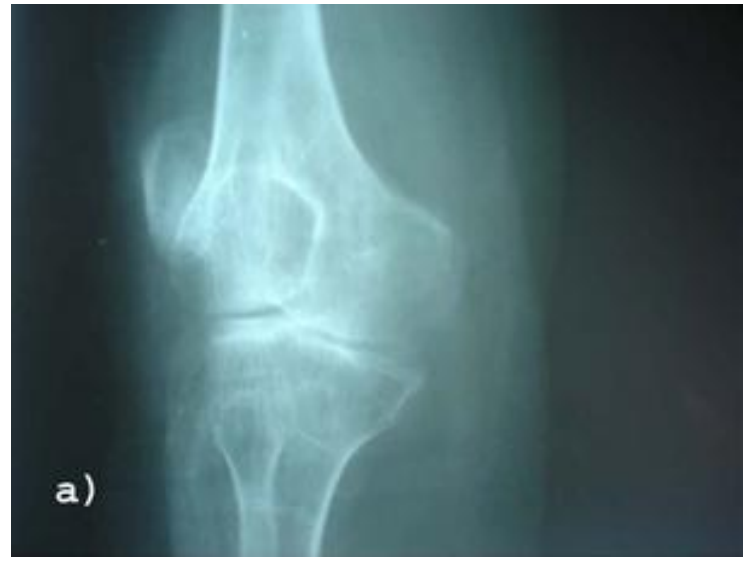

Figure 2a

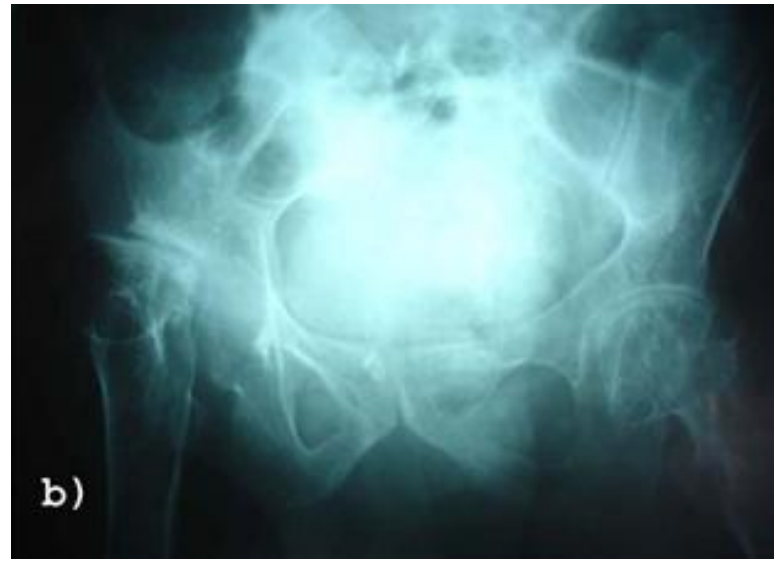

Figure $2 b$ 


\section{CASE REPORT}

Lymphocyte infiltrate in muscle biopsy - Hematoxylin and Eosin stain.

3a): Lymphocytic infiltration surrounding atrophied muscles and fibrous band (40x)

3b): Magnified view show the clear infiltration of lymphocytes around the muscle fibers (100x).

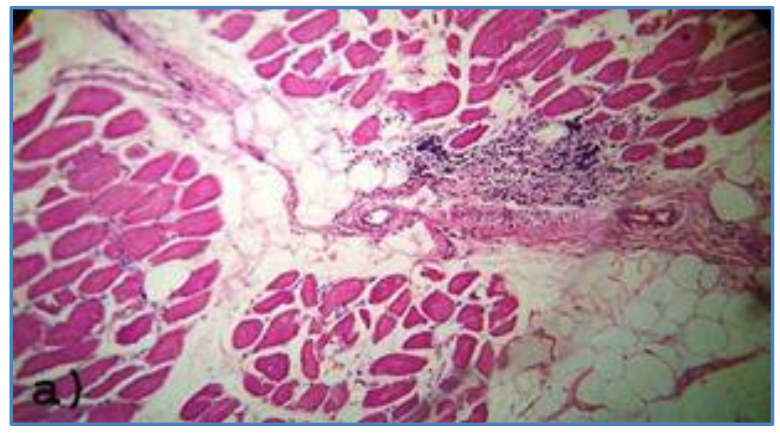

Figure 3a

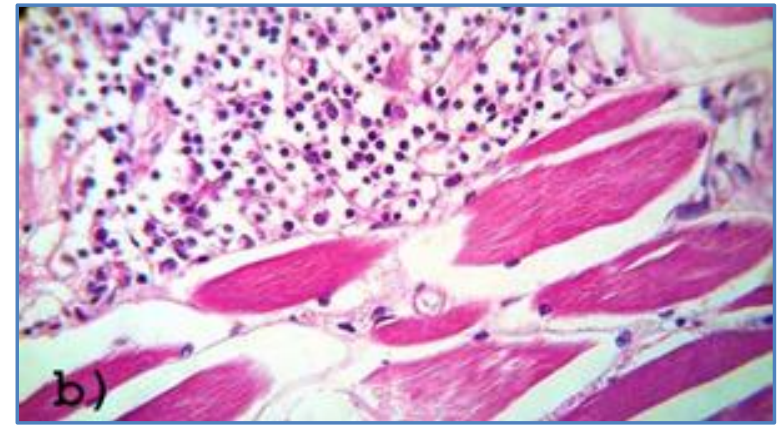

Figure 3b

Fibrosis and Granuloma. Hematoxylin and Eosin ( $40 \mathrm{x}$ )

4a): Muscle fibers interspersed with excessive fibrous bands.

4b): Granuloma formation seen inside the muscle fibers.

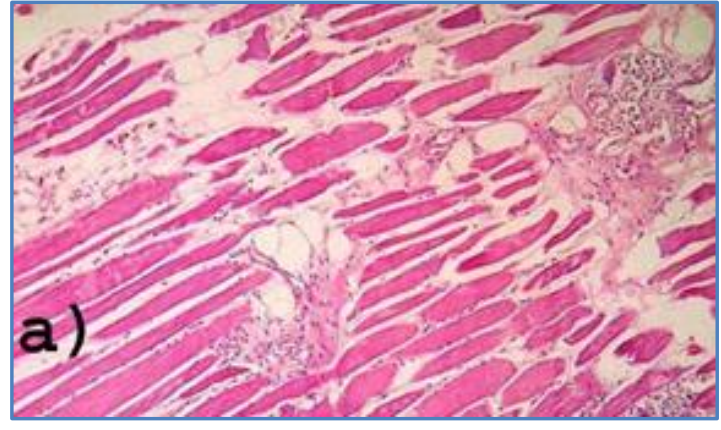

Figure 4a

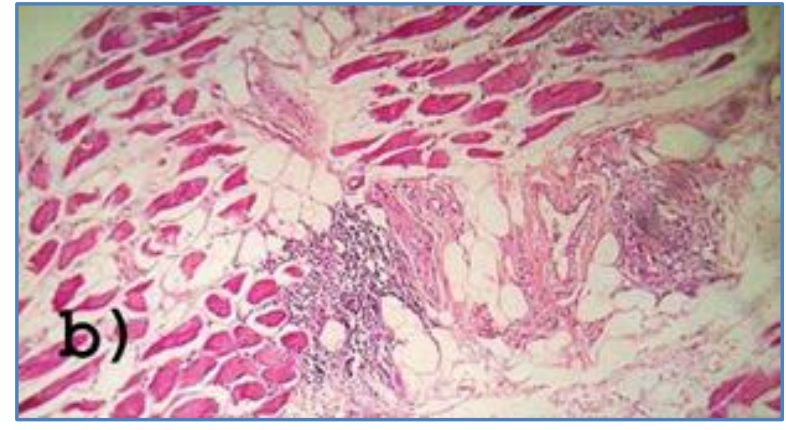

Figure $4 b$

\section{AUTHORS:}

1. Sundar S.

2. Muthuraj K.

3. Nagarajan $\mathrm{V}$.

4. Ameen Azad

\section{PARTICULARS OF CONTRIBUTORS:}

1. Assistant Professor, Department of Neurology, SRM Medical College.

2. Professor and HOD, Department of Neurology, SRM Medical College.

3. Associate Professor, Department of Neurology, SRM Medical College.

4. Senior Resident, Department of Neurology, SRM Medical College.

\section{NAME ADDRESS EMAIL ID OF THE} CORRESPONDING AUTHOR:

Sundar Shanmugam,

Assistant Professor,

Department of Neurology,

SRM Medical College \& Research Centre,

Kattankulathur, Chennai-603203.

Email: drradnus@gmail.com

Date of Submission: 07/07/2014.

Date of Peer Review: 08/07/2014.

Date of Acceptance: 21/07/2014.

Date of Publishing: 22/07/2014. 